\title{
Active memory processes in visual sentence comprehension: Clause effects and pronominal reference
}

\author{
FREDERICK R. CHANG \\ University of Oregon, Eugene, Oregon 97403
}

\begin{abstract}
Two experiments were conducted to investigate active memory processes during reading. Subjects read two-clause sentences one word at a time at a self-paced rate. Following each sentence a probe word was presented; subjects were to decide if this word occurred in the sentence they had just read. The first experiment examined clausal effects during reading. Reaction times to items from the final clause were shorter than those to items from a previous clause even when the same number of words intervened. The second experiment used the clause effect to address the issue of proniminal reference. Results indicated that a pronoun in the final clause activated the meaning of its antecedent, thus demonstrating that the method is sensitive to both surface and meaning codes in active memory.
\end{abstract}

The complex process through which readers turn printed symbols on a page into meanings has been the subject of much experimentation in both psychology and education. The present study investigates the role of active memory as one aspect of this comprehension process. The usage of the term "active memory" should be viewed as similar to that of "working memory" (cf. Baddeley \& Hitch, 1974) or "operational memory" (cf. Posner, 1973) in the sense that it represents a control system with limits on both storage and processing capabilities. The aim of the present research is essentially twofold: To investigate the function of active memory during reading, and to develop a method through which the process can reliably be studied. Two experiments are reported here. The first investigated how active memory might be influenced by clausal boundaries in sentences, and it served to test and validate the method used. The second experiment employed this method to examine the issue of pronominal reference.

Most of the research looking at active memory processes for linguistic materials has investigated the processing of auditorily presented stimuli. One of the major theories of sentence processing involving a con-

This paper is based on a thesis submitted to the University of Oregon in partial fulfillment of the requirements for the Master of Arts degree. Portions of this research were presented at the meeting of the Western Psychological Association in San Diego, California, on April 6, 1979. I would like to express my appreciation to Michael Posner for his support and guidance throughout this project. I am also grateful to Albert Corbett, Wayne Wickelgren, and Douglas Hintzman for their advice and comments on this research. This work was supported by Grant NIE-G-77-0008 from the National Institute of Education while the author held Public Health Service Traineeship 5-T01GM02165 granted to the University of Oregon. Requests for reprints should be sent to Frederick R. Chang, Department of Psychology, University of Oregon, Eugene, Oregon 97403. sideration of active memory has been advanced by Bever and his colleagues (e.g., Bever, Lackner, \& Kirk, 1969; Fodor \& Bever, 1965; see also Fodor, Bever, \& Garrett, 1974, for a review of much of this literature). According to the theory, clauses are viewed as the basic units of encoding in active memory. Thus, sentence processing is presumed to occur in a clause-by-clause manner, with detailed information from a currently processed clause available in a readily accessible form. However, once a clause has been assigned a semantic analysis, this detailed information is no longer as accessible.

There has been some experimental evidence supporting this contention. Jarvella (1971) presented listeners with short stories with instructions for comprehension. $\mathrm{He}$ interrupted the subjects at various points in the stories, and their task was to write down as much as they could remember verbatim. The results were that subjects' rote recall was best for the material in the clause immediately preceding the interruption and fell off markedly for material that occurred prior to that clause. He found a similar effect for sentence boundaries. Jarvella concluded that both clauses and sentences serve as units in language comprehension, and he suggested that meaning is extracted in a clause-by-clause manner and that a clause is dismissed from immediate memory as soon as its meaning has been extracted.

Further evidence for this notion has been provided by Caplan (1972) using an item-recognition task in the context of speech recognition. In this study, listeners heard two-clause sentences such as:

(1) Now that artists are working in oil / prints are rare.

(2) Now that artists are working fewer hours / oil prints are rare. 
The slash $(/)$ indicates a clause boundary. Notice that in both sentences, the final four words ("oil prints are rare") are identical. The grammatical structure of each sentence determines whether "oil" is in the initial clause (Sentence 1) of the sentence or in the final clause (Sentence 2). One hundred milliseconds following the offset of the sentence, subjects were presented a probe word (e.g., "oil"), and reaction time (RT) was measured for their decision regarding whether or not the probe word occurred in the sentence immediately preceding it. The results were that $\mathrm{RT}$ to the probe word was longer in sentences like Sentence 1 than in sentences like Sentence 2. Again, this provides evidence that clauses are processed as units, with information about the individual lexical items that occurred in the clause avail. able in an accessible form as it is being processed.

In the area of reading, there is some evidence from research on the eye-voice span that clauses act as units (e.g., Levin \& Kaplan, 1970). In a typical study of this kind, a subject is instructed to read a section of text aloud; then, at certain critical points, the text is removed from view. The subject is instructed to report as much in advance of the interruption as is possible. The extent to which the eye leads the voice provides a measure of segmentation in reading. It has been found that when the text was removed from view at a point when the subject's voice was close to a clause boundary, the size of the eye-voice span tended to be smaller than when the text was removed from view when the subject's voice was further from the boundary.

Finally, in an experiment involving the measurement of subjects' reading times, Aaronson and Scarborough (1976) also demonstrated an influence of grammatical structure. With instructions for either memorization or comprehension, readers in this study pressed a button to read each successive word in sentences, and reading time for each word was recorded. For the readers in the memorization condition, reading times were increased at clause boundaries and bowed within a clause. The pattern of reading times for subjects in the comprehension condition showed no such influence of syntactic structure. Instead, the times reflected more of an influence due to the semantic content of the sentence.

The method used in the experiments reported here is essentially a hybrid of the procedures used by Aaronson and Scarborough (1976) and Caplan (1972). Readers paced themselves through sentences one word at a time, depressing a button to receive each successive word. Reading times for each individual word were measured. Following the offset of the final word in the sentence, subjects immediately received a single probe word and were to decide whether or not that word occurred in the sentence immediately preceding it. Choice RT was measured. Following this old-new judgment, subjects received a yes-no comprehension question.

Of primary importance is the RT to the old-new judgment. The key assumption is this: With regard to "old" responses, RTs should be faster to words (or concepts) that are more accessible or active in memory than to words that are no longer as accessible. A related item-recognition task has been used by Kennedy (1975) in a study on associative priming; more recently, Ratcliff and McKoon (1978) employed a similar procedure to find evidence for the propositional structure of sentences in memory. The data collected on the time subjects spend reading individual words in sentences could provide evidence about certain on line reading processes.

Subjects can make the old-new judgment on the basis of two distinct levels of information (cf. Posner, Lewis, $\&$ Conrad, 1972). Matches can be made on the basis of surface information, that is, whether a particular letter string occurred in the sentence. Matches can also be made through the use of meaning information, where a response is made due to the fact that a certain concept occurred in the sentence. Thus, this method can poten. tially examine the activation of both types of informa. tion in reading.

Given the sizable amount of evidence that clauses act as units in sentence processing, Experiment 1 is designed to validate the method and to extend the Caplan (1972). result into the domain of visual sentence comprehension. Thus, the predictions are that probe words occurring in the final clause of two-clause sentences will be verified more quickly than probe words from the initial clause. An additional aim of this experiment was to investigate how these clause effects might vary as a function of the nature of the materials used. This experiment employed a set of carefully controlled (and thus somewhat con. trived) sentences such as Sentences 1 and 2 above and a set of more naturalistic sentences. It is of some interest to know whether the anticipated clause effects are similar for the two types of materials. If not, this might suggest that readers adopt a differential processing strategy for the different types of sentences.

\section{EXPERIMENT 1}

\section{Method}

Stimulus sentences and design. Two types of two-clause sentences were used in this experiment: "controlled" and "natural." The controlled sentences were adapted from Caplan (1972) and were similar in form to Sentences 1 and 2 above. Each pair consisted of one sentence in which the target item (e.g., "oil") occurred in the initial clause of the sentence (as in Sentence 1) or in the final clause of the sentence (as in Sentence 2), depending on the particular syntactic construction. There were 12 sentence pairs, and each member of a pair was assigned to one of two lists. Each list contained an equal number of sentences in which the target word appeared in the initial clause and sentences in which it appeared in the final clause.

These sentences control many factors exactly: serial position effects, the actual target and probe word tested, and even the particular lexical items that occurred between target and test. However, they contain local ambiguities and are identical in syntactic form (subordinate clause/main clause construction). It is desirable to extend the generality of the comparison to a broader class of sentences. Thus, another set of test sentences consisting of natural sentences was selected from a variety of 
sources, including the Reader's Digest, Time, Newsweek, and the like. Examples are given below:

(3) When the wind began to get stronger in the early afternoon / the sun warmed us.

(4) As midsummer nears and the pond begins to shrink / frogs crawl into the mud.

The slash $(/)$ indicates a clause boundary, and the italicized words are the target items. These sentences included subordinate clause/main clause constructions and main clause/subordinate clause constructions, as well as sentences with coordinate clause structures. As in the controlled sentences, serial position effects were controlled by insuring that the same number of intervening items occurred between target and test for sentences in which the target word occurred in the initial clause compared with when it occurred in the final clause. These natural sentences confounded probe items with the grammatical structure manipulation, but they were free of some of the problems found in the controlled sentences. The mean number of words occurring between target and test for both types of sentences was 5.0 $(\mathrm{SD}=1.7)$

The design of the experiment was a four-way factorial. Target location (target word occurs in either the initial or final clause) and sentence type (controlled or natural) were both repeated measures; subjects were nested within orders (three different random orders of presentation were used). There were six observations per cell of the design.

In addition to the test sentences, there were 30 distractor sentences. These sentences, like the natural sentences, were selected from a number of popular magazines and comprised a variety of syntactic structures. Eighteen were probed with a synonym of some element of the sentence and thus required a "new" response. The other 12 required "old" responses, but a word at a clause boundary was not probed.

Subjects. Twelve undergraduate students served as subjects in this experiment. They were obtained from the subject pool for the Cognitive Laboratory at the University of Oregon and were paid $\$ 2.50$ for their participation in the 35 -min session. All subjects were native Engish speakers and had normal or corrected-to-normal vision.

Procedure. Prior to each trial in the experiment, the word "READY" appeared in the center of a cathode-ray tube display. Then subjects read a sentence, one word at a time. A three-key keyboard was used such that the subjects could rest their thumbs on the middle key and their index fingers on the outer keys of the keyboard. Subjects depressed the middle key to read each successive word in the sentence, and the individual word reading times were recorded. Immediately following the keypress to the final word in the sentence, subjects were presented a probe word. They were to decide, using their index fingers on the outer keys, whether or not this word occurred in the sentence immediately preceding it. An "old" response was made with the left hand, and a "new" response was made with the right hand. RT to this decision was measured. In order to make the probe word clearly distinguishable from the words in the sentence, it was presented 2 deg lower on the screen. The subjects' responses terminated the probe word; $500 \mathrm{msec}$ later, subjects were presented a yes-no comprehension question, and they indicated their responses again with their index fingers on the outer keys, with a "yes" response on the left and a "no" response on the right. The "questions" were actually presented in statement form. This eliminated the interrogative terms, thus minimizing the number of words. Following the question, subjects were given feedback (for $2 \mathrm{sec}$ ) on the speed and accuracy of their comprehension question responses. This was done to encourage good comprehension. This feedback terminated each trial.

Subjects were instructed to read the sentences as quickly as they could while maintaining good comprehension. They were also instructed to respond as quickly and accurately as possible to both the probe word and the comprehension question. Subjects sat $60 \mathrm{~cm}$ from the screen, and a typical word subtended roughly $3 \mathrm{deg}$ of visual angle. A PDP-15 laboratory computer controlled all aspects of the experiment.

\section{Results and Discussion}

Old-new judgment. Of primary importance in this experiment are the results of the old-new judgment. It was predicted that if subjects process sentences in a clause-by-clause manner in reading, then they should be faster and more accurate in responding to probe words occurring in the final clause of a sentence than in the initial clause. Further, it is of interest to note whether the effect is consistent over sentence types (controlled vs. natural). The RT and error results are presented in Table 1. Subjects were indeed faster at responding to probe words that occurred in the final clause of sentences than to those that occurred in the initial clause. This effect was reliable $[F(1,9)=14.51, p<.01, \mathrm{MSe}=$ $3,960]$. Moreover, the effect was identical over sentence types, as there was no main effect of this variable or an interaction. The main effect of orders was significant $[F(2,9)=4.75, p<.05, \mathrm{MSe}=76,290]$, but it did not interact with any of the other variables. A similar pattern of results was found in the error data. Subjects were more accurate at responding to probe words occurring in the final clause than to those in the initial clause. This error result was reliable $[F(1,9)=5.65$, $p<.05, \quad M S e=66]$. No other effects approached significance.

Reading times. It is of interest to know if subjects paused at clause boundaries as they read the sentences because, if they did, then there would be evidence for on-line effects of grammatical structure as subjects were trying to comprehend the sentences. Pausing at clause boundaries might cause a different interpretation of the results from the old-new judgment. If subjects spent longer with final words in the initial clause than with initial words in the final ciause, then the two words would not be equated for presentation duration, and this could influence performance on the old-new judgment. Reading times were virtually flat as a function of the clause boundary. This was confirmed by a one-way analysis of variance treating number of words before or after the clause boundary as a factor $(\mathrm{F}<1, \mathrm{MSe}=957)$. Moreover, there was no evidence of pausing due to semantic content, as the reading time difference between

Table 1

Mean Reaction Times (RT) in Milliseconds and Percent Errors (PE) for Experiment 1

\begin{tabular}{llcrr}
\hline & \multicolumn{4}{c}{ Target Location } \\
\cline { 2 - 5 } Sentence & \multicolumn{2}{c}{ Initial Clause } & \multicolumn{2}{c}{ Final Clause } \\
\cline { 2 - 5 } Type & RT & PE & RT & PE \\
\hline Controlled & 847 & 7.0 & 790 & 2.8 \\
Natural & 879 & 7.0 & 797 & .0 \\
Mean & 863 & 7.0 & 794 & 1.4 \\
\hline
\end{tabular}


Table 2

Mean Reading Times (in Milliseconds) as a Function of Serial Position for Experiment 1

\begin{tabular}{|c|c|c|c|c|c|c|c|c|c|c|c|c|c|c|c|c|c|c|}
\hline & \multicolumn{18}{|c|}{ Serial Position } \\
\hline & 1 & 2 & 3 & 4 & 5 & 6 & 7 & 8 & 9 & 10 & 11 & 12 & 13 & 14 & 15 & 16 & 17 & $18+$ \\
\hline Reading Tìme & 430 & 374 & 370 & 393 & 382 & 378 & 382 & 381 & 386 & 398 & 389 & 391 & 406 & 409 & 402 & 419 & 409 & 416 \\
\hline Sentences* & 54 & 54 & 54 & 54 & 54 & 54 & 54 & 54 & 54 & 53 & 49 & 46 & 41 & 31 & 24 & 22 & 16 & 33 \\
\hline
\end{tabular}

*Number of sentences that went into each reading time.

content and function words did not approach significance.

One interesting result that did emerge from these data was found when reading times were plotted as a function of serial position. These data are presented in Table 2. Disregarding the first serial position (i.e., the first word in each sentence), there was a consistent linear increase in reading time as the subjects advanced through the sentences. A least-squares linear-regression line was fit to the data. The slope $(2.66 \mathrm{msec})$ and fit $(r=.90)$ were significantly different from zero $(p<.01)$. The intercept of the line was 365 msec. Precisely the same pattern of results was evidenced for the controlled, natural, and distractor sentences separately. This linear increase might reflect the integration of each successive word into the sentence. Since each new word adds information to the sentence, slightly more time is required for integration.

The most important result from this experiment is evidence for clause boundary effects in active memory for written materials. This supports the notion that detailed information from a currently processed clause is in a more readily accessible form than information from a previous clause. The effect was identical for both contrived and natural sentences.

The method used in this experiment can be used to investigate other differences in coding between active memory for the final clause read and active memory for the previous clause. One such issue involves pronominal reference. This was the focus of Experiment 2.

\section{EXPERIMENT 2}

Readers in Experiment 1 could perform the old-new judgment based solely on surface (including syntactic) information. Since the goal in reading is the apprehension of meanings, it is important to study the role of meaning in active memory. This can be accomplished through the use of pronominalization. A pronoun must in some way reinstate or activate the meaning representation of its anaphor if comprehension is to occur.

The issue of pronominal reference is a small but essential component of the comprehension process that has been given increased consideration recently as cognitive scientists have attempted to understand the process of comprehension. In the field of artificial intelligence, there have been attempts to incorporate pronominal reference into language understanding systems (Norman \& Rumelhart, 1975; Winograd, 1972).
The few empirical studies that have been performed have largely been concerned with characterizing the nature of the search process people go through in locating pronominal antecedents (e.g., Clark \& Sengul, 1979; Springston, 1975). The present experiment investigated the extent to which a simple pronoun reinstates or activates the meaning representation of its referent and studied difference in coding between a currently processed clause and a prior one.

The result in Experiment 1 in conjunction with pronominalization can be used to examine these issues. Consider the following examples:

(5) John and Mary went to the grocery store / and John bought a quart of milk.

(6) John and Mary went to the grocery store / and he bought a quart of milk.

The slash $(/)$ indicates a clause boundary. In Sentence 5, the word "John" in the second clause refers, of course, to the word "John" in the first clause. However, in Sentence 6, the word "he" in the second clause also refers unambiguously to "John" in the first clause. It is of interest to know how the simple pronoun "he" serves to reinstate the meaning representation for "John." Suppose subjects, after reading Sentence 5 or 6 , are required to decide whether or not the probe word "John" occurred in the sentence. The response, of course, is "yes" (or "old"). Considering the information available in the second clause (this is the currently processed clause at the time subjects make a response) of either sentence, subjects can make their responses in Sentence 5 on the basis of surface information, because the letter string "John" has just been presented in the second clause. However, in Sentence 6 this is not true; if subjects use information from the second clause, then they must use meaning information to make their responses, because the word "he" refers to the meaning representation for "John." If subjects do not or cannot use meaning information in active memory, then they must make their responses to Sentence 6 on the basis of information from the first clause.

Of course, a control condition is needed in which subjects can use only information from the first clause to make their responses. Consider the following:

(7) John and Mary went to the grocery store / and (Mary) (she) bought a quart of milk. 
The slash (/) indicates a clause boundary. Again, as in Sentences 5 and 6, suppose subjects are required to decide if the probe word "John" occurred, after they have read Sentence 7 . In this case they cannot use any information in the second clause to assist in a response; they must use information from the first clause. Since information from the first clause is not as readily accessible, their responses should be delayed compared with when Sentence 5 is read. This prediction is based on the results from Experiment 1. Thus, responses to the probe word "John" should be faster and more accurate in Sentence 5 than in Sentence 7. If the pronoun "he" in Sentence 6 is every bit as effective as the word "John" in reinstating the meaning representation for "John," and subjects use only this meaning information for a response, then responses to the probe word "John" in Sentence 6 should be just as fast and accurate as those in Sentence 5. However, if "he" serves to reinstate the concept of "John" not at all or subjects do not make use of meaning information in active memory, then responses to the probe word "John" in Sentence 6 should be equal to those in Sentence 7, the control condition. There is also the possibility of the intermediate case, in which response time and accuracy to the probe word in Sentence 6 would fall somewhere in between those for Sentences 5 and 7.

\section{Method}

Stimulus sentences and design. Each test sentence used in this experiment had a compound agent in the first clause consisting of a male and a female proper name, followed by either a name (male or female) of a third person pronoun (male or female) in the second clause. The name or pronoun referred unambiguously to one member of the compound agent in the first clause. The probe word following each test sentence was always one of the male or female proper names occurring in the first clause. The probe word could refer either to the same person as that in the second clause (reinstated case) or to a person mentioned only in the first clause (control case). Some examples will make this clearer. Consider Sentences 5, 6, and 7 once again, and assume that the probe word following each sentence is "John." Sentences 5 and 6 illustrate the reinstated cases. In Sentence 5, "John" occurs in the second clause, and since the probe word is also "John," the probe word refers to both the letter string and the concept presented in the second clause. This is the reinstated name condition. In Sentence 6, "he" occurs in the second clause and, of course, refers to "John." Since the probe word is "John," again it refers to the same concept, but not to the letter string in the second clause. This is the reinstated pronoun condition. Sentence 7 illustrates the control conditions. Here, "Mary" or "she" is read in the second clause, but since the probe word is "John," it refers to information presented only in the first clause. Thus, when "Mary" is presented in the second clause, this is the control name condition; when "she" is presented in the second clause, this is the control pronoun condition.

Forty-eight quadruples of test sentences were generated. For example, there was a basic sentence like Sentence 5, and then "John," "he," "Mary," or "she" occurred in the second clause of the variation sentences. Each member of a quadruple was assigned to one of four lists. Each list contained an equal number of sentences in each of the four conditions. Thus, there were 12 test sentences in each of the four conditions in each list. The male or female proper name occurred in the first serial position equally often (i.e., "John and Mary" or "Mary and John" occurred equally often) in each list. In constructing the 48 basic sentences, four different conjunctions were used: "and," "but," "so," and "when." Each conjunction was used in 12 sentences in each of the lists. Finally, the male and female proper names were taken from the Battig and Montague (1969) production norms. The male and female names that made up the compound agent of each sentence were matched closely in terms of production frequency. Each proper name was used only once, and across the entire experiment male and female names were used equally often as probe words. The mean number of words occurring between target and test was $5.1(\mathrm{SD}=1.3)$.

The design of the experiment was a four-way factorial. Reinstatement (reinstated or control) and second clause subject (name or pronoun) were both repeated measures; subjects were nested within lists (of which there were four). There were 12 observations per cell of the design.

In addition to the test sentences (which all required an "old" response), there were 84 distractor sentences. Forty-two also included a use of male and female proper names, but they varied in form. That is, some were compound agent sentences like the test sentences, and some were subject-object sentences, in which the male or female name could appear as subject or object. Of these 42,24 tested a false (or "new") name, 6 tested a false verb, 6 tested a true verb, and 6 tested a true object. In addition, there were 42 "natural" sentences, similar in form to those used in Experiment 1. Of the 42,14 required an "old" response (various elements of the sentences were tested), and 28 required a "new" response (synonyms of sentence elements were probed).

Subjects. Sixteen students obtained from the subject pool for the Cognitive Laboratory at the University of Oregon served as subjects in this experiment. All were native speakers of English and had normal or corrected-to-normal vision. They were paid $\$ 2.50$ for their participation in the 45 -min session.

Procedure. The procedure used in this experiment was identical to that used in Experiment 1. Following the experiment, each subject was queried about reading a high proportion of sentences with names and pronouns. Subjects mentioned that they noticed it, but they reported that it did not affect the way they normally read in any fundamental way (i.e., adopting some idiosyncratic strategy of coding names only).

\section{Results}

Old-new judgment. Of main importance in this experiment are the RT and error results for the old-new judgment. These data are presented in Table 3. An analysis of variance was performed on the means of the RT data. This analysis revealed a large main effect of reinstatement; that is, the reinstated conditions were faster than the control conditions $[F(1,12)=21.13$, $\mathrm{p}<.001, \mathrm{MSe}=2,492]$. More important, however, there was a significant Reinstatement by Second Clause Subject interaction $[F(1,12)=5.97, \mathrm{p}<.05, \mathrm{MSe}=$ $1,472]$. A planned comparison, breaking down this

Table 3

Mean Reaction Times (RT) in Milliseconds and Percent Errors (PE) for Experiment 2

\begin{tabular}{cccccc}
\hline & \multicolumn{4}{c}{ Reinstatement } \\
\cline { 2 - 3 } Second Clause & \multicolumn{2}{c}{ Reinstated } & & \multicolumn{2}{c}{ Control } \\
\cline { 2 - 3 } \cline { 5 - 6 } Subject & RT & PE & & RT & PE \\
\hline Name & 694 & 1.6 & & 775 & 4.7 \\
Pronoun & 743 & 2.1 & & 777 & 4.7 \\
\hline
\end{tabular}


interaction, revealed that the reinstated name condition was significantly faster than the reinstated pronoun condition $\quad[F(1,15)=6.33, \quad p<.025, \quad \mathrm{MSe}=3,036]$ and that the reinstated pronoun condition was significantly faster than the mean of the control name and control pronoun conditions $[\mathrm{F}(1,15)=6.77, \mathrm{p}<.025$, $\mathrm{MSe}=1,717]$. The control name and control pronoun conditions did not significantly differ $(\mathrm{F}<1, \mathrm{MSe}=$ 3,465 ), and there was no main effect of second clause subject. The three-way List by Reinstatement by Second Clause Subject interaction just did make significance $[F(3,12)=3.61, p=.046, \mathrm{MSe}=1,472]$. This means merely that the Reinstatement by Second Clause Subject interaction varied slightly through the four lists.

The error data revealed precisely the same pattern of results; however, the effects were not as strong, as only the main effect of reinstatement was significant (the reinstated name and reinstated pronoun conditions were responded to more accurately than the control name and control pronoun conditions) $[\mathrm{F}(1,12)=6.59, \mathrm{p}<.025$, $\mathrm{MSe}=20]$.

Reading times. The pattern of reading times in this experiment was identical to that found in Experiment 1 . There was no evidence of pausing at clausal boundaries. This was verified by a one-way analysis of variance treating number of words before and after the clause boundary as a factor $(\mathrm{F}<1, \mathrm{MSe}=729)$. Additionally, as in Experiment 1, there was no evidence of pausing due to semantic content, as the reading time difference for content and function words did not approach significance.

As in Experiment 1, there was a marked linear increase in reading times as the subjects read through the sentences (disregarding the first serial position). These data are presented in Table 4. A least-squares linear-regression line was fit to the data. The slope $(2.63 \mathrm{msec})$ and fit $(\mathrm{r}=.87)$ were significantly different from zero $(p<.01)$. The intercept of the line was $287 \mathrm{msec}$. Precisely the same pattern was found for the test and distractor sentences separately.

One potentially interesting reading time result is the time subjects take to read a name or pronoun in the second clause. It is possible that when reading a pronoun in the second clause, subjects pause in locating its antecedent, compared with when a name is read, when such an anaphoric search is not necessary. The results indicate that there were slightly longer reading times for pronouns compared with proper names (306 $\mathrm{msec}$ for pronouns in the second clause vs. $299 \mathrm{msec}$ for names), but this difference did not approach significance $[F(1,15)$ $=1.33, \mathrm{MSe}=316]$. Perhaps the subjects did not make the anaphoric search on-line or perhaps an anaphoric search is required for a name as well as for a pronoun. In any event, it may be that the effects were small and the reading time measure was not sensitive enough to pick them up. This seems likely, given the absence of syntactic and semantic effects in reading time in both experiments.

\section{Discussion}

The results from the old-new judgment in this experiment clearly demonstrate that the method is sensitive to both surface and meaning codes in active memory. That a meaning code is present is evidenced by the fact that the probe word was verified more quickly and accurately in the reinstated pronoun condition than in the control conditions. Thus the pronoun served to reinstate the meaning representation of its referent and facilitated probe-verification performance relative to the control conditions. The "meaning" of a proper name in this experiment presumably involves constructing a representation for a human of a particular sex. The presence of a surface code is demonstrated by the fact that the probe word in the reinstated name condition was verified more quickly and accurately than in the reinstated pronoun condition. This advantage is presumably due to the fact that the probe word in the reinstated name condition matched the target word in both meaning and surface form, while only meaning was matched in the reinstated pronoun condition. This additional surface-level information thus facilitated performance.

The difference between the reinstated name and reinstated pronoun conditions is evidently not due to the pronoun's being more difficult to process. If so, then the control name and control pronoun conditions would be expected to differ in a similar manner. However, this was not the case, as performance in the two conditions was virtually identical.

A slightly different account of the results involves the notion of partial activation. That is, the reason that the RTs and errors for the reinstated pronoun condition fell in between the reinstated name and control conditions could be some sort of partial reinstatement of the concept by the pronoun. In this view, the name in the reinstated name condition served to totally reinstate the meaning representation of the appropriate referent, while the pronoun in the reinstated pronoun condition

Table 4

Mean Reading Times (in Milliseconds) as a function of Serial Position for Experiment 2

Serial Position

\begin{tabular}{|c|c|c|c|c|c|c|c|c|c|c|c|c|c|c|c|c|}
\hline & 1 & 2 & 3 & 4 & 5 & 6 & 7 & 8 & 9 & 10 & 11 & 12 & 13 & 14 & 15 & $16+$ \\
\hline Reading Time & 360 & 302 & 295 & 295 & 299 & 300 & 298 & 299 & 303 & 303 & 309 & 312 & 316 & 328 & 322 & 342 \\
\hline Sentences* & 132 & 132 & 132 & 132 & 132 & 132 & 132 & 132 & 132 & 132 & 130 & 124 & 106 & 84 & 53 & 64 \\
\hline
\end{tabular}

*Number of sentences that went into each reading time. 
did so to a lesser degree. However, since the sentences used in this experiment were quite simple and the referent of the pronoun was completely unambiguous, the pronoun might have been expected to fully reinstate the meaning representation of its antecedent. Thus, the results seem better stated in terms of the surface and meaning codes distinction. Since the very nature of the old-new judgment requires that the subject decide whether or not a certain visually identical letter string occurred before, the task should be influenced by the surface information present only in the reinstated name condition.

The reading time data from the present experiments are apparently in conflict with the data from the comprehension subjects in the Aaronson and Scarborough (1976) study. Their study found pausing at content words, whereas the present study did not. Moreover, a linear increase in reading time over serial positions was found here, but not in their study. Two important differences between the studies include the task and the stimulus sentences. The task required in this study included probe recognition followed by a comprehension question, whereas the Aaronson and Scarborough (1976) study required only the comprehension question. Additionally, the stimulus sentences employed here were, in general, simpler than those used in their study. Merely pointing out the differences, of course, provides no answers, but at this point an explanation would be somewhat speculative.

In conclusion, the goal to develop a method to reliably study active memory processes in reading has apparently been realized. The fact that the method is sensitive to both surface and meaning codes suggests that both aspects of the reading process could be studied. More generally, it is of some interest to know whether having subjects read one word at a time influenced their normal comprehension process in some fundamental way. While this research was not designed to answer this question, there is some relevant literature. The work by Rayner and McConkie (1977) on the size of the perceptual span in reading indicates that meaning information is extracted from a relatively narrow region around the point of fixation. This region corresponds to roughly 1.5 to 2 words. Information about word shape and word length is extracted from the area beyond this narrow band. However, comprehension is evidently unaffected by the absence of this information. An experiment by Patberg and Yonas (1978) presented readers with either normal text or text that permitted a perceptual span of only one word (there were 13 letter spaces between words). For skilled readers, they found that, while the one-word presentation slowed reading speed, comprehension was unaffected compared with normal text. Thus, there is some reason to think that the one-word presentation used in these experi- ments did not influence readers' normal comprehension processes and that this method could be used to elucidate important characteristics of the reading process.

\section{REFERENCES}

Aaronson, D., \& Scarborough, H. S. Performance theories for sentence coding: Some quantitative evidence. Journal of Experimental Psychology: Human Perception and Performance, 1976, 2, 56-70.

BadDElEY, A. D., \& Hitch, G. Working memory. In G. H. Bower (Ed.), The psychology of learning and motivation (Vol. 8). New York: Academic Press, 1974.

Battig, W. F., \& Montague, W. E. Category norms for verbal items in 56 categories: A replication and extension of the Connecticut category norms. Journal of Experimental Psychology Monograph, 1969, 80(3, Pt. 2).

Bever, T. G., Lackner, J. R., \& Kirk, R. The underlying structures of sentences are the primary units of immediate speech processing. Perception \& Psychophysics, 1969, 5, 225-231.

Caplan, D. Clause boundaries and recognition latencies for words in sentences. Perception \& Psychophysics, 1972, 12, 73-76.

Clark, H. H., \& Sengul, C. J. In search of referents for nouns and pronouns. Memory \& Cognition, 1979, 7, 35-41.

Fodor, J. A., \& Bever, T. G. The psychological reality of linguistic segments. Journal of Verbal Learning and Verbal Behavior, 1965, 4, 414-420.

Fodor, J. A., Bever, T. G., \& Garrett, M. F. The psychology of language. New York: McGraw-Hill, 1974.

J ARVELLA, R. J. Syntactic processing of connected speech. Journal of Verbal Learning and Verbal Behavior, 1971, 10, 409-416.

Kennedy, A. Contextual effects in reading and recognition. In A. Kennedy \& A. Wilkes (Eds.), Studies in long term memory. New York: Wiley, 1975.

LEVIN, H., \& KaPLAN, E. L. Grammatical structure in reading. In $\mathrm{H}$. Levin \& J. P. Williams (Eds.), Basic studies on reading. New York: Basic Books, 1970.

Norman, D. A., \& Rumelhart, D. E. Explorations in cognition. San Francisco: Freeman, 1975.

Patberg, J. P., \& Yonas, A. The effects of the reader's skill and the difficulty of the text on the perceptual span in reading. Journal of Experimental Psychology: Human Perception and Performance, 1978, 4, 545-552.

Posner, M. I. Cognition: An introduction. Glenview, Ill: Scott, Foresman, 1973.

Posner, M. I., Lewis, J. L., \& Conrad, C. Component processes in reading: A performance analysis. In J. Kavanaugh \& I. Mattigly (Eds.), Language by ear and by eye. Cambridge, Mass: M.I.T. Press, 1972.

Ratcliff, R., \& MCKoon, G. Priming in item recognition: Evidence for the propositional structure of sentences. Journal of Verbal Learning and Verbal Behavior, 1978, 17, 403-417.

Rayner, K., \& McConkiE, G. W. Perceptual processes in reading: The perceptual spans. In A. Reber \& D. Scarborough (Eds.), Toward a psychology of reading. Hillsdale, N.J: Erlbaum, 1977.

Springston, F. J. Some cognitive aspects of presupposed coreferential anaphora. Unpublished doctoral dissertation, Stanford University, 1975.

Winograd, T. Understanding natural language. New York: Academic Press, 1972.

(Received for publication May 15, 1979; revision accepted September $28,1979$. 